\title{
Flora inventory of Köprülü Kanyon National Park (Antalya-Isparta)
}

\author{
Hasan Özçelik*
}

\begin{abstract}
In this study, vascular flora of Köprülü Kanyon National Park had been investigated. Results according to identification of samples are as follows: 230 vascular endemics of the area and their conservation status were published previously. Pteridophyta 16, Gymnospermae 14, Magnoliopsida 595 and Liliopsida 82, totally 707 (950 if included its environs) taxa have determined. About 150 of these taxa are new registrations for square $\mathrm{C} 3$. The distributional rates of the taxa according to phytogeographic regions: Mediterranean 219 (30.97\%), Irano-Turanian 74 (10.46 \%) and Euro-Siberian 50 (07.07 \%). Vascular plant list of the natural park and its environs are reported. Genera: 8 Pteridophyta, 6 Gymnospermae, 318 Dicotyledoneae, 42 Monocotyledoneae (totally 374 genera); Familias: 8 Pteridophyta, 3 Gymnospermae, 76 Dicotyledoneae, 8 Monocotyledoneae(totally 95 families). The families and genera which including the most taxa: Families: Lamiaceae (Labiatae) 87 (12.30\%), Fabaceae (Leguminosae) 78 (11.03\%), Asteraceae (Compositae) 71 (10.04\%), Brassicaceae (Cruciferae) 47 (6.64 \%), Scrophulariaceae 39 (5.51\%), Rosaceae 35 (4.95\%), Liliaceae 34 (4.80\%), Ranunculaceae 33 (\% $4.66 \%)$, Boraginaceae 32 (4.52 \%), Apiaceae (Umbelliferae) 31 (4.38\%) and Caryophyllaceae 28 (3.96 \%). Genera: Ranunculus 18 (2.54 \%), Veronica and Geranium 13 (1.83), Vicia 12 (1.69), Trifolium 11 (1.55 \%), Rosa, Verbascum and Sedum 10 (1.41), Galium 9 (1.27), Euphorbia and Stachys 8 (1.13), Sideritis,Hypericum, Trigonella and Astragalus 7 (0.99 \%), for each Lathyrus, Thymus, Micromeria and Silene $6(0.84 \%)$. Total taxa in dangerous: 48, endemic taxa in dangerous in the park: 44, total endemic taxa: 230; local endemic taxa number for the area and near surroundings: 19, total vascular plant taxa: 707 (950 all together near fields). By Bern Convention: A1 (Number of globally threatened species): 5, A2 (Number of endangered species in European scale): 39. C2 (Number of threatened habitats): 6 (42.A17, 421951, 42B12, 42.6643, 4285B1, 45.11).
\end{abstract}

Keywords: Flora, Köprülü Kanyon National Park, Biodiversity, Systematics, Biogeography

\section{Köprülü Kanyon Milli Parkı'nın (Antalya-Isparta) flora envanteri}

\begin{abstract}
Özet: Bu çalışmada, Köprülü Kanyon Milli Parkı'nın ve çevresinin vasküler florası araştırılmıştır. Toplanan örneklerin yapılan teşhislerinin sonucuna göre sonuçlar aşağıdaki gibidir: Alandan 230 endemik iletim demetli bitki taksonu ve koruma statüleri daha önce yayınlandı. Bu makalede, vasküler(damarlı) bitkilerin listesi rapor edilmiş̦tir. Cins sayıları: 8 Pteridophyta 6 Gymnospermae, 318 Dicoyyledoneae, 42 Monocotyledoneae, genel toplam 374); Familyalar: 8 Pteridophyta, 3 Gymnospermae, 76 Dicotyledoneae, 8 Monocotyledoneae, genel toplam 95. Pteridophyta 16, Gymnospermae 14, Magnoliopsida 595 and Liliopsida 82, toplam olarak 707 (çevresiyle birlikte 950) takson ihtiva eder. Bu taksonlardan yaklaşık 150'si C3 karesi için yeni kayıttır. Taksonların fitocoğrafi bölgelere dağılımı ve dağılım oranları şöyledir: Akdeniz 219 (\% 30.97), Iran-Turan 74 (\% 10.46) ve Avrupa-Sibirya 50 (\% 07.07). Endemik taksonların sayıs1 230 olup toplam floraya oranı \% 24.21'tür. Cins sayısı: Pteridophyta 8, Gymnospaermae 6, Dicoyledoneae 318 ve Monocotyledoneae 42 (toplam olarak 374); Familyalar: Pteridophyta 8, Gymnospaermae 3, Dicoyyledoneae 76 ve Monocotyledoneae 8 (toplam olarak 95). En çok takson içeren familya ve cinsler şunlardır: Familyalar: Lamiaceae (Labiatae) 87 (\% 12.30), Fabaceae (Leguminosae) 78 (\% 11.03), Asteraceae (Compositae) 71 (\% 10.04), Brassicaceae (Cruciferae) 47 (\% 6.64), Scrophulariaceae 39 (\% 5.51), Rosaceae 35 (\% 4.95), Liliaceae 34 (\% 4.80), Ranunculaceae 33 (\% $4.66 \%$ ), Boraginaceae 32 (\% 4.52), Apiaceae (Umbelliferae) 31 (\% 4.38) ve Caryophyllaceae 28 (\% 3.96) Cinsler: Ranunculus 18 (\% 2.54), Veronica and Geranium 13 (\% 1.83), Vicia 12 (\% 1.69), Trifolium 11 (1.55\%), Rosa, Verbascum and Sedum 10 (1,41 \%), Galium 9 (1.27), Euphorbia and Stachys 8 (\% 1.13), Sideritis, Hypericum, Trigonella and Astragalus 7 (\% 0.99), Lathyrus, Thymus, Micromeria and Silene için 6 (\% 0.84). Tehlikedeki takson sayısı: 48, Milli Parkta tehlikede endemik takson sayıs1: 44, toplam endemic takson sayıs1: 230; çalışma alanı ve çevresine özgü endemik takson sayısı: 19. Bern Sözleşmesine göre: A1 (Küresel Ölçekte tehlike altındaki habitatlar): 39. C2 (Tehlike altındaki habitatlar): 6 (42.A17, 421951, 42B12, 42.6643, 4285B1, 45.11).
\end{abstract}

Anahtar kelimeler: Flora, Köprülü Kanyon Milli Parkı, Biyoçeşitlilik, Sistematik, Biyocoğrafya

\section{Introduction}

Türkiye has a distinctly unique and rich flora in the neighbouring countries because of situated on the crossroads of three phytogeographical regions; also its vegetation history, different climatical, topographical conditions etc. In this sense, it is like an open-air museum. It is one of a few rich countries in terms of endemic plants, their percentage rate being about $35 \%$. The country is also being rich in monotypical genera and endemic taxa. The country is also the birthplace of some cultural plants and the gene centre of some genera like Astragalus, Verbascum etc. Lakes District may be a gene centre for the genera Silene and Rosa and Bolanthus (Özçelik, 1995; 2000).

Unfortunately, over the last 50 years these natural richness of the country have been threatened by over-

\footnotetext{
$\triangle$ a Süleyman Demirel University, Science and Letter Faculty, Department of Biology, Isparta

@* Corresponding author (İletişi̇m yazarı): hasanozcelik@ sdu.edu.tr

$\checkmark \quad$ Received (Geliş tarihi): 19.09.2017, Accepted (Kabul tarihi): 14.03.2018
}

Citation (Atıf): Özçelik, H., 2018. Flora inventory of Köprülü Kanyon National Park (Antalya-Isparta). Turkish Journal of Forestry, 19(1): $40-50$ DOI: $10.18182 /$ tjf.338944 
pasturing, grazing, moving, fires, cutting, construction, urbanization, industrialization and antropogenic factors like herbicide using. As a result of the above mentioned reasons while some endemic and rare plants are undergoing a substantial decline in numbers, others are being destroyed (Küçüker, 1995). To bring the problems to light, a list of threat categories for rare and endemic plants has been made. According to this list, 12 endemic species are extinct (Ekim et.al., 2000).

The area is located in Sütçüler (Isparta), Manavgat and Serik (Antalya) districts; mostly in Antalya province. The area according to the Davis's grid system is in the C3 square. The area is located in $37^{\circ} 17^{\prime} \mathrm{N}$ and $31^{\circ} 06^{\prime} \mathrm{E}$. The people of the region earn their lives from the production of farm animals and agricultural plant cultivations in general. Animals kept for production were small ruminants and have been grazing on the pastures from the spring to the beginning of winter. The geological and geomorphologic status of the canyon have revealed a natural wonder when it is integrated with the richness of regional flora, and the whole area has been named as a National Park in $12^{\text {th }}$ December, 1973. It has total area of 36. 614 ha and administrated by the Antalya-Isparta Directorate of Nature Protection and National Parks (Özçelik, 2012).

The mountain range in which the study area is located is very important for the Lakes Region (Figure 1). Flora of the region is very rich, vast and generally in a natural form. And it is also rich in economical importance plants especially medicinal. This area begins around Beşkonak village of National Park with the area of $130(-110) \mathrm{m}$ altitude and up to the peak of Bozburun mountain at $2505 \mathrm{~m}$. Main land is generally in a conglomerate structure which can be found in flat land of alluvial and river beds. The total length of the canyon is about $14 \mathrm{~km}$. The slope of hill sides has a $90 \%$ inclination in some places. As a whole, Köprülü Kanyon National Park has the Mediterranean climatic type. However, in high altitudes, a continental climate is dominant especially in borders Konya and Isparta (Özçelik, 2012). There are importance of the topographic and floristic structures of this area in the formation of the microclimate of the region. Therefore the formation of agricultural biodiversity in the region, the use of plants for various purposes, the using cultures and their trades due to the research area. The area is the gene center of Rosa dumalis subsp. boissieri var. antalyensis. The Mediterranean region is also Rosaceae, Apiaceae and Lamiaceae. It is very rich in terms of fruit trees. This has been a major factor in the development of fruit agriculture in the region. It is one of the 4 sections under the GEF II Project.

For the reasons, the essential aims of the study are to determine and protect the biological diversity of the area. It is to continue generations of rare and endemic plants in the area. Three 3 important aims of the project by us are:
1. To determine and protect important plants (generations of the endemics, rare and economics etc.) and to work various ecosystems in the park at first step,

2. To reduce up to minimum degree negative effects of human (local people and visitors etc.) on the area and its environs by means of a good management strategy of the national park,

3. To supply new financial sources for local people and to strengthen the socio-economical situation.

In the paper, it is aimed only to give vascular plant list of the national park. Other results of the project will be published in near time.

\section{Material and method}

In respect to growing period of plants, the Köprülü Kanyon National Park has been surveyed since April 2003. Floristic inventory studies were occured from specialists in different work areas. Nearly 2000 plant samples are collected from the area. Only native or naturalized plants have been collected from the area. Almost 50 specimens couldn't be identified. In general, as a good rule of thumb is to collect only a small set of samples from a large population. It was been avoided collecting of abundant plant samples for each taxon. Only a few samples of a species in flower or fruit collected from the local habitat for scientific identification and the show room of the National Park visitors. The voucher herbarium samples beloging to vascular plants which formed basic of our research material, were dried, labelled and written corresponding to the rules. In identifying of the samples, it was been mostly used from Flora of Turkey and East Aegean Islands (Davis, 19651985; Güner et al., 2000). The specimens have been collected from various localities of the park in the years 2003-2004. The samples of dried vascular plants deposited in the GUL Herbarium at S. Demirel University (Isparta). But in vascular plant list in the paper, only plant taxa could being reported. In all account of it approximately gives following plant list. In the text, under headings pteridophytes, gymnosperms, dicots and monocots, vascular plants are listed alphabetically in the sequence of families, but genera, species rows and numbered according to Flora of Turkey and the East Aegean Islands written by Davis (1965-1985). It was shortly discused after floristical lists. New record taxa for C3 square were determined in the light of the references (Donner, 1985; Huber-Morath, 1987; 1988; Nydegger-Hügli, 1994; Özçelik and Korkmaz, 2002; Özhatay et al., 2005; Serin, 1984; Fakir, 2006a; 2006b).

Abbreviations in the text:

* : New record taxon for C3 square,

** : Plant has economic importance in the region,

Cult : Cultivated in the research area or cultured in general. 

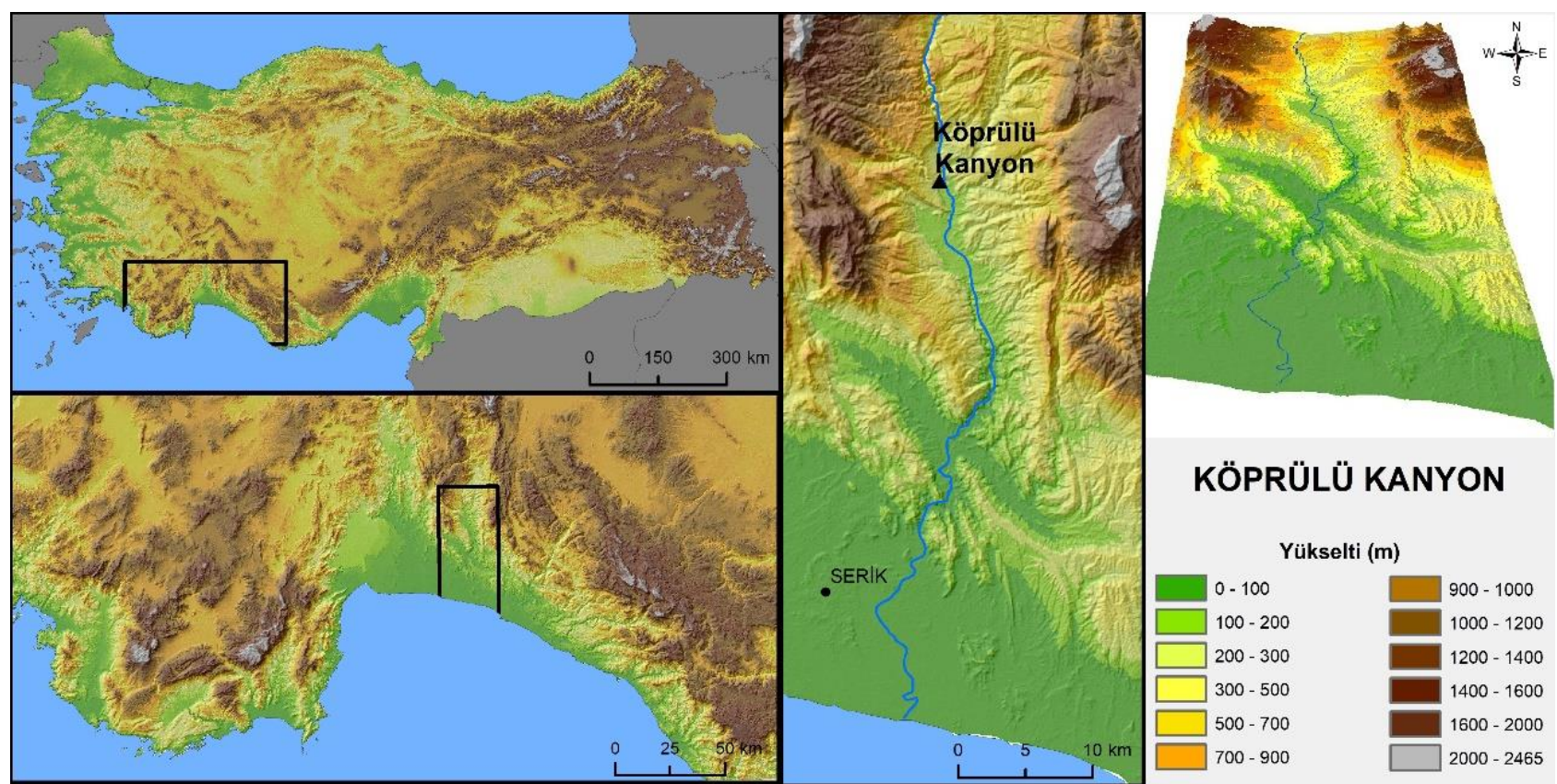

KÖPRÜLÜ KANYON

Yükselti (m)

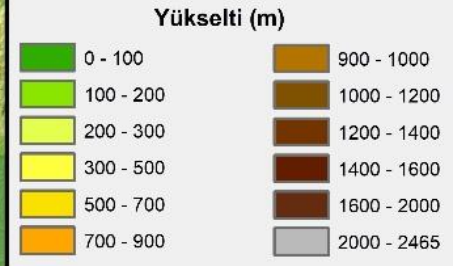

Figure 1. View from satellite of research area (https://earth.google.com/web).

3. Results (Vascular plants of the study area)

\section{Division 1: Pteridophyta}

\section{Adiantaceae}

Adiantum capillus-veneris L.**

\section{Aspidiaceae}

Dryopteris filix-mas (L.) Schott**, D. pallida (Bory) Fomin subsp. pallida**, D. pallida (Bory) Fomin

\section{Aspleniaceae}

Asplenium trichomonas L., A. bourgaei Millde, A. onopteris L., A. ceterach L. subsp. ceterach**.

\section{Athyriaceae}

Cystopteris fragilis (L.) Bernh**

\section{Equisetaceae}

**Equisetum hyemale L.*, E. ramosissimum Desf.**, $* *$ E. arvense L.*

Hypolepiadaceae

Pteridium aqulinum (L.) Kuhn**

Selaginellaceae

Selaginella denticulata (L.) Link

\section{Sinopteridaceae} Kuhn

Cheilanthes fragrans (L. fil.) Sw., C. persica (Bory)

\section{Division 2: Spermatophyta}

\section{Subdivision 1: Gymnospermae}

Cupressaceae
Cupressus sempervirens L. var. horizontalis Mill.**, $C$. s. cv. "pyramidalis"**, Juniperus oxycedrus L. subsp. oxycedrus**, J. foetidissima Willd.**, J. sabina L.*, J. excelsa Bieb.**

\section{Pinaceae}

Abies cilicica (Ant. \& Kotschy) Carr. subsp. isaurica Coode \& Cullen.**, Cedrus libani A. Rich.**, Pinus nigra Arn. subsp. pallasiana (Lamb.) Holmboe**, P. brutia Ten**, P. pinea L.**

\section{Ephedraceae \\ **Ephedra major Host*, **E. campylopoda C.A.} Meyer*

\section{Subdivision 2: Angiospermae}

\section{Classis 1: Magnoliopsıda /Dicotyledoneae}

Acanthaceae

Acanthus hirsutus Boiss.**, A. spinosus L.**

Aceraceae

Acer monspessulanum L. subsp. monspessulanum**, A.m. subsp. microphyllum (Boiss.) Bornm.*, A. negundo L.*

Anacardiaceae

**Cotinus coggyria Scop.*, Rhus coriaria L.**, Pistacia lentiscus L.**, P. terebinthus L. subsp. palaestina (Boiss.) Engler**

\section{Apiaceae (Umbelliferae)}

Astrantia maxima Pallas subsp. haradjianii (Grintz.) Rech. fil.**, Eryngium campestre L.**, Lagoecia cuminioides L., Echinophora tournefortii Jaub. \& Spach.**, Scandix iberica M. Bieb., S. pecten-veneris L., S. australis L. subsp. grandiflora (L.) Thell., S. turgida (Boiss. \& Bal.) Boiss., Bunium microcarpum (Boiss.) Freyn subsp. 
bourgaei (Boiss.) Hedge \& Lamond, **Pimpinella corymbosa Boiss.*, **Conium maculatum L.*, Lecokia cretica (Lam.) DC.*, Bupleurum croceum Fenzl. B. odontites L.*, B. sulphureum Boiss. \& Bal., B. anatolicum Hub.- Mor. \& Reese*, Falcaria vulgaris Bernh.*, Malabaila secacul Banks \& Sol.**, **Heracleum pastinacifolium C. Koch*, Tordylium apulum L., $T$. brachytaenum Boiss. \& Heldr, T. pestalozzae Boiss.* South, Ainsworthia trachycarpa Boiss.*, **Glaucosciadium cordifolium (Boiss.) Brutt \& Davis*, Laserpitium petrophilum Boiss. \& Heldr.**, Torilis arvensis (Huds.) Link subsp. elongata (Hoffmans. \& Link) Cannon, T. leptophylla (L.) Reichb., Caucalis platycarpos L., Turgenia latifolia (L.) Hoffm, Daucus guttatus Sm.

\section{Apocynaceae}

Nerium oleander L.**

Araliaceae

Hedera helix L.**

Aristolochiaceae

**Aristolochia clematitis L.*, A. maurorum L.**, A. bodamae Dingler*

Asclepiadaceae

Cynanchum acutum L. subsp acutum, Vincetoxicum canescens (Willd.) Decne subsp. pedunculata Browicz*, Cionura erecta (L.) Griseb.*

\section{Asteraceae (Compositae)}

Xanthium spinosum L.*, **Inula orientalis Lam.*, I. anatolica Boiss.**, I. graveolens (L.) Desf., I. viscosa (L.) Aiton**, Pulicaria dysenterica (L.) Bernh.**, Helichrysum pamphylicum Davis \& Kupicha**, H. stoechas (L.) Moench subsp. barrelieri (Ten.) Nyman**, H. chasmolycicum P H. Davis, H. compactum Boiss.*, H. plicatum DC. subsp. plicatum**, Filago vulgaris Lam*, Bellis perennis L.**, Senecio vulgaris L.*, S. vernalis Waldst. \& Kit., Tussilago farfara L.**, **Calendula officinalis L.*, C. arvensis L., Anthemis rosea Sm. subsp. carnea (Boiss.) Grierson**, Achillea wilhelmsii C. Koch.**, **A. vermicularis Trin.*, **A. nobilis L. subsp. sipylea (O. Scwarz) Bassler**, Artemisia absinthium L.*, A. campestris L.*, Arctium minus (Hill.) Bernh. subsp. pubens (Babiryeton) Arénes*, Onopordum anatolicum (Boiss.) Eig.**, Silybium marianum (L.) Gaertner*, Cirsium libanoticum DC. subsp. lycaonicum (Boiss. \& Heldr.) Davis \& Parris, C. arvense (L.) Scop., Picnomon acarna (L.) Cass.**, Ptilostemon afer (Jacq.) Greuter subsp. eburneus Greuter, Carduus nutans L.**, Centaurea virgata Lam., C. calolepis Boiss., C. solstitialis L. subsp. carneola (Boiss.) Wagenitz, C. iberica Trev. ex Sprengel, C. depressa Bieb., Crupina vulgaris Cass.*, C. crupinastrum (Moris) Vis., Cnicus benedictus L. var. kotschyi Boiss.**, Carthamus lanatus L.**, C. dentatus Vahl.**, Xeranthemum annuum L.**, Echinops ritro L., E. pannosus Rech. fil.*, E. viscosus DC. subsp. bithynicus (Boiss.) Rech. fil., E. orientalis Trautv.*, Scolymus hispanicus L.**, Cichorium intybus L.**, Scorzonera cana (C.A. Meyer) Hoffm. var. radicosa (Boiss.) Chamberlain**, Tragopogon longirostris Bisch. ex. Schultz Bip.**, T. dubius Scop.**, T. pratensis L.*, T. buphthalmoides (DC.) Boiss. var. buphthalmoides*, T. aureus Boiss.*, Picris strigosa Bieb., Sonchus asper (L.) Hill.**, S. oleraceus L.**, Pilosella hoppeana (Schultes) C.H. \& F.W. Schultz, P. piloselloides (Vill.) Sojak. subsp. piloselloides, P. p. subsp. megalomastix (NP.) Sell \& West, Lactuca serriola L.*, Lapsana communis L., Taraxacum syriacum Boiss.*, Chondrilla juncea L. var. juncea**, Crepis foetida L.subsp. rhoeadifolia (Bieb.) Celak., C. sancta (L.) Babcock.

Berberidaceae

Berberis crataegina DC.**

\section{Boraginaceae}

Heliotropium bovei Boiss.*, H. europaeum L.*, Rochelia disperma (L. fil.) C. Koch subsp. disperma, Myosotis incrassata Guss., M. ramosissima Rochel ex Schultes subsp. ramosissima*, Omphalodes ripleyana Davis**, Paracaryum cappadocicum Boiss. \& Bal., Solenanthus stamineus (Desf.) Wettst.**, Cynoglossum creticum Miller, Lithospermum officinale L.*, $L$. purpurocaeruleum L.*, Buglossoides arvensis (L.) Joksten, Echium italicum L.**, Moltkia coerulea (Wild.) Lehm., M. aurea Boiss., Onosma gracile Trautv.*, Cerinthe glabra Miller*, Symphytum orientale L.*, Anchusa leptophylla Roemer \& Schultes subsp. tomentosa (Boiss.) Chamb.*, A. officinalis L.*, A. undulata L. subsp. hybrida (Ten.) Coutinho.**, A. limbata Boiss. \& Heldr., A. aggregata Lehm., Nonea rosea (Bieb.) Link*, N. vesicolor (Steven) Sweet*, N. anchusoides Boiss. \& Buhse*, Alkanna tinctoria (L.) Tausch. subsp. anatolica Hub.-Mor.**, A. t. subsp. subleiocarpa (Hub.-Mor.) Hub.-Mor., A. areolata Boiss. var. areolata**, A. areolata Boiss. var. subleavis Hub.Mor.*, A. oreodaxa Hub.-Mor., A. punctulata Hub-Mor.*

Brassicaceae (Cruciferae)

**Sinapis alba L.*, S. arvensis L.**, Hirschfeldia incana (L.) Lag.-Foss, Diplotaxis tenuifolia (L.) DC.**, Conringia orientalis (L.) Andrz.*, Cardaria draba (L.) Desv. subsp. chalepensis (L.) O.E. Schultz*, Isatis cappadocica Desv. subsp. alyssifolia (Boiss.) Davis**, Iberis attica Jord.*, Biscutella didyma L.**, Aethionema arabicum (L.) Andrz. ex DC.**, Thlaspi perfoliatum L., T. alliaceum L.*, Capsella bursa-pastoris (L.) Medik.**, Neslia apiculata Fisch., Mey. \& Ave-Lall., Fibigia clypeolata (L.) Medik., F. macrocarpa (Boiss.) Boiss.*, Alyssum dasycarpum Steph ex Willd.*, A. desertorum Stapf. var. desertorum, A. minus (L.) Rothm. var. minus, A. lepidotum Boiss., A. condensatum Boiss. \& Hausskn subsp. condensatum, A. filiforme Nyar*, Clypeola jonthlaspi L., Erophila verna (L.) Chevall., Arabis andosacea Fenzl*, A. deflexa Boiss., A. ionocalyx Boiss., A. caucasica Willd. subsp. brevifolia (DC.) Cullen, A. abietina Bornm*, A. nova Vill., Turritis glabra L.*, T. laxa (Sibth. \& Sm.) Hayek*, Nasturtium officinale R.Br.**, Barbarea verna (Mill.) Aschrts., Cardamine graeca L., Aubrieta canescens (Boiss.) Bornm. subsp. canescens, A. pinardii Boiss., Matthiola longipetala (Vent.) DC. subsp. bicornis (Sibth. \& Smith) P.W. Ball.**, Malcolmia africana (L.) R.Br.*, M. chia (L.) DC., M. flexuosa (Sibth. \& Sm.) Sibth. \& Sm., M. micrantha Boiss. \& Reut.*, M. crenulata (DC.) Boiss.*, Erysimum leucanthemum (Steph.) Fedtsch.*, Alliaria petiolata (Bieb.) Cavara \& Grande*, Sisymbrium officinale (L.) Scop.**, S. altissimum L.*, Descurainia sophila (L.) 
Webb ex Prantl, Camelina hispida Boiss. var. grandiflora (Boiss.) Hedge.

\author{
Capparaceae \\ Capparis ovata Desf.**, C. spinosa L.** \\ Caprifoliaceae \\ **Lonicera etrusca Santi var. etrusca*.
}

\section{Campanulaceae}

Campanula iconia Phitos.*, C. lyrata Lam. subsp. lyrata, C. cymbalaria Sm., Asyneuma linifolium (Boiss. \& Heldr.) Bornm. subsp. linifolium, A. michauxioides (Boiss.) Dambold., Legousia falcata (Ten.) Fritsch**, L. pentagonia (L.) Thellung**.

\section{Caryophyllaceae}

Arenaria deflexa Dec. subsp. microsepala McNeill, A. pamphylica Boiss. \& Heldr. subsp. pamphylica var. turcica McNeill, A. sabulinea Gris. ex Fenzl*, A. serpyllifolia L., Minuartia glandulosa (Boiss. \& Huet.) Bornm*, M. umbellulifera (Boiss.) McNeill subsp. umbellulifera var. umbellulifera, C. macranthum Boiss.*, Holosteum umbellatum L. var. umbellatum*, Moenchia mantica (L.) Bartl. subsp. mantica*, Bufonia calyculata Boiss. \& Bal.**, B. tenuifolia L.*, Spergularia bocconii (Scheele) Aschers. \& Graebn., Telephium imperati L. subsp. orientale ( Boiss.) Nyman**, Dianthus spp.**, Petrorhagia hispidula (Boiss. \& Heldr.) Ball \& Heywood, Velezia rigida L., $V$. pseudorigida Hub.-Mor., **Saponaria syriaca Boiss.*, $S$. kotschyi Boiss.**, Vaccaria pyramidata Medik. var. pyramidata*, Silene spergulifolia (Desf.) Bieb., S. vulgaris (Moench.) Garcke var. macrocarpa (Turrill) Coode \& Cullen**, S. leptoclada Boiss., S. flavescens Waldst. \& Kit.*, S. balansae Boiss.*, S. colorata Poiret**, Agrostemma githago L.**

\section{Chenopodiaceae}

**Chenopodium botrys L.*, C. foliosum (Moench) Aschers**, C. album L. subsp. album var. album**

\section{Cistaceae}

Cistus creticus L.**, C. salviifolius L.**, **C. laurifolius L.*, Fumana arabica (L.) Spach. var. arabica, Tuberaria guttata (L.) Fourr. var. clandestina (Vierh.) Davis $\&$ Coode

\section{Convolvulaceae}

Convolvulus dorycnium L. subsp. oxysepalus (Boiss.) Rech.*, C. scammonia L.**, **Ipomoea purpurea (L.) Roth.*

\section{Cornaceae \\ Cornus mas L.**}

\section{Crassulaceae}

Umbilicus erectus DC., U. rupestris (Salisb.) Dandy, $U$. horizontalis (Guss.) DC. var. horizontalis, Rosularia serpentinica (Wendermann) Muirhead**, Sedum sediforme (Jacq.) Pau, S. amplexicaule DC., S. acre L.*, S. lydium Boiss.*, S. cepaea L.*, S. annuum L.*, S. caespitosum (Cav.) DC., $S$. hispanicum L. var. hispanicum*, $S$. hispanicum L. var. planifolium Chamberlain*, S. rubens L.**

$$
\begin{aligned}
& \text { Cucurbitaceae } \\
& * * \text { Ecballium elaterium (L.) A. Rich.*, Bryonia cretica } \\
& \text { L.**,**B. alba L.* }
\end{aligned}
$$

\section{Cuscutaceae \\ Cuscuta campestris Yuncker*.}

Dipsacaceae

Dipsacus laciniatus L.**, Cephalaria lycica Matthews*, Scabiosa micrantha Desf.*, S. persica Boiss.*, S. rotata Bieb., Tremastelma palaestinum (L.) Janchen*

$$
\begin{aligned}
& \text { Elaeagnaceae } \\
& \text { **Elaeagnus angustifolia L.* }
\end{aligned}
$$

\section{Ericaceae}

Erica manipuliflora Salisb. **, Arbutus andrachne L.**

\section{Euphorbiaceae}

Chrozophora tinctoria (L.) Rafin.**, Euphorbia peplis L., E. dendroides L.*, E. apios L., E. peplus L. var. peplus, E. falcata L. subsp. macrostegia (Bornm.) O. Schwarz, E. rigida Bieb., E. lucida Waldst. \& Kit.*, E. characias L. subsp. wulfenii (Hoppe ex W. Koch) A.R. Smith.

Fabaceae (Leguminosae)

Ceratonia siliqua L.**, Cercis siliquastrum L. subsp. hebecarpa (Bornm.) Yalt.**, Acacia retinoides Schlecht*, Chamaecytisus eriocarpus (Boiss.) Rothm.**, Gonocytisus angulatus (L.) Spach, Genista burdurensis P.Gibbs*, G. aucheri Boiss.*, Spartium junceum L., Calicotome villosa (Poiret) Link, Lotononis genistoides (Fenzl) Benth.**, **Lupinus albus L. subsp. albus*, L. a. subsp. graecus (Boiss. \& Sprun.) Franco \& Silva*, **Colutea cilicica Boiss. \& Bal.*, C. melanocalyx Boiss. \& Heldr. subsp. melanocalyx**, C. m. subsp. davisiana (Browicz) Chamb.*, Astragalus suberosus Banks \& Sol. subsp. haarbachii (Sprun.) Matthews**, A. panduratus Bunge*, A. echinops Aucher ex Boiss.*, A. lycius Boiss., A. gaeobotrys Boiss. \& Bal.*, A. schizopterus Boiss., A. barbarae Bornm.*, Psoralea bituminosa L.**, P. jaubertina Fenzl*, Vicia freyniana Bornm*, V. cracca L. subsp. atroviolaceae (Bornm.) Davis*, V. cracca L. subsp. stenophylla Vel., V. villosa Roth. subsp. villosa, $V$. v. subsp. eriocarpa (Hausskn.) P.W. Ball**, V. cassia Boiss., V. pubescens (DC.) Link., V. peregrina L., V. lutea L. var. lutea (Balbis) Lois.**, V. pannonica Crantz var. purpurascens (DC.) Ser.*, V. sativa L. subsp. sativa**, V. narbonensis L.**, Lens montbretii (Fisch. \& Mey) Davis \& Plitm.*, L. nigriscans (Bieb.) Godr.*, L. ervoides (Brign.) Grande**, Lathyrus digitatus (Bieb.) Fiori, L. laxiflorus (Desf.) Kuntze subsp. laxiflorus*, L. setifolius L., L. gorgoni Parl. var. gorgoni, L. aphaca L. var. aphaca*, Pisum sativum L. subsp. elatius (Bieb) Aschers \& Graebn. var. elatius**, Ononis adenotricha Boiss. var. adenotrica**, O. natrix L. subsp. hispanica (L. fil.) Coutinho, O. pubescens L., $O$. pusilla L., O. spinosa L. subsp. leiosperma (Boiss.) Sirj.**, **Trifolium repens L. var. repens*, T. $r$. var. giganteum Lag-Foss*, T. campestre Schreb.**, T. physodes Stev. ex Bieb. var. physodes, T. resupinatum L. var. microcephalum 
Zoh.*, T. g. var. nervulosum (Boiss. \& Heldr.) Zoh., T. pratense L. var. pratense*, T. medium L. var. medium*, $T$. sylvaticum Guard ex Lois., T. arvense L. var. arvense, $T$. angustifolium L. var. angustifolium ${ }^{* *}$, Melilotus indica (L.) All., M. officinalis (L.) Desr., **Trigonella balansae Boiss. \& Reuter*, T. spinosa L.*, T. fischeriana Ser., $M$. orbicularis (L.) Bart.**, $M$. sativa L. subsp. sativa**, **M. x. varia Martyn*, M. minima (L.) Bart. var. minima, $M$. polymorpha $\mathrm{L}$. var. polymorpha, Lotus corniculatus L.. var. alpinus Ser.**, Anthyllis vulneraria L. subsp. praepropera (Kerner) Bornm.*, A. tetraphylla L., Securigera securidaca (L.) Degen. \& Dörf., Coronilla emerus L. subsp. emeroides (Boiss. \& Sprun) Uhrova**, C. grandiflora Boiss., Ornithopus L., O. compressus L., Scorpiurus muricatus L. var. subvillosus (L.) Fiori, Onobrychis ptolemaica (Del.) DC.*

\section{Fagaceae}

Castanea sativa Miller**, **Quercus robur L. subsp. pedunculiflora (C. Koch) Menitsky*, Q. infectoria Olivier subsp. boissieri (Reuter) O. Schwarz**, Q. cerris L. var. cerris $^{* *}, * * Q$. libani Olivier*, $Q$. coccifera L.**

\section{Fumariaceae}

Corydalis wendelboi Liden subsp. wendelboi**, $* * C$. solida (L.) Clairv. subps. incisa Liden*, C. rutifolia (Sibth. \& Sm. ) DC., $* * C$. erdelii (Zacc.) Cullen \& Davis* $C$. conorhiza Ledeb.*, Fumaria capreolata L., $* * F$. microcarpa Boiss. ex Hausskn.*, F. parviflora Lam.**, **F. asepala Boiss.*

\section{Gentianaceae}

Centaurium erythraea Rafin subsp. erythraea*, C. e. subsp. rumelicum (Velen) Melderis*, C. spicatum (L.) Fritsch, C. maritimum (L.) Fritsch*

\section{Geraniaceae}

Geranium lucidum L.**, G. purpureum Vill.**, G. rotundifolium L.**, G. molle L. subsp. molle**, G. m. L. subsp. brutium (Gasp.) Davis*, G. divaricatum Ehrh., G. columbinum L., G. tuberosum L. subsp. tuberosum**, **G. stepporum Davis*, G. macrostyllum Boiss.*, $G$. asphodeloides Burm. fil. subsp. asphodeloides*, $G$. pyrenaicum Burm. fil., G. ibericum Cav. subsp. jubatum

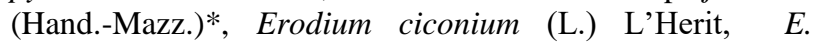
moschatum (L.) L'Herit, E. acaule (L.) Bech erer \& Thell.*, Pelargonium endlicherianum Fenzl.**

\section{Globulariaceae}

Globularia trichosantha Fisch. \& Mey.**

\section{Hamamelidaceae}

Liquidambar orientalis Miller var. integriloba Fiori**

\section{Hypericaceae}

Hypericum ternatum Poulter**, $H$. scabrum L., $H$. hirsutum L.*, **H. venustum Fenzl*, $H$. lanuginosum Lam. var. lanuginosum**, H. l. var. scabrellum (Boiss.) Robson*, H. cuisii Barbey*, H. aviculariifolium Jaub. \& Spach subsp. depilatum (Freyn \& Bornm.) Robson var. depilatum.
Illecebraceae

Herniaria glabra L.**, H. micrantha A.K. Jackson \& Turrill*, H. incana Lam.**, Paronychia argentea Lam. var.

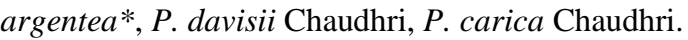

Juglandaceae

Juglans regia L.**

Labiatae (Lamiaceae)

Ajuga chamaepitys (L.) Schreber subsp. cuneifolia (Stapf) P.H. Davis**, A. bombycina Boiss.**, Teucrium chamaedrys L. subsp. chamaedrys**, T. c. subsp. lydium O. Schwarz**, T. divaricatum Sieber subsp. villosum (Celak.) Rech. fil., T. montanum L., T. polium L.** ${ }_{2}$. lamiifolium d'Urv. subsp. lamiifolium**, **Rosmarinus officinalis L.*, Scutellaria megalaspis Rech. fil.**, **S. orientalis L. subsp. orientalis*, **Phlomis lunariifolia $\mathrm{Sm}$., P. grandiflora H.S. Thompson**, P. longifolia Boiss.\& Bl. var. bailanica (Vierh.) Hub.-Mor.*, P. armeniaca Willd.**, P. kurdica Rech. fil.*, Lamium pisidicum R. Mill., L. eriocephalum Bentham. subsp. glandulosidens (Hub.-Mor.) R. Mill*, $L$. amplexicaule L., L. purpureum L. var. aznavorii Gand. ex Aznov., L. album L.*, Ballota cristata P.H. Davis**, **B. nigra L. subsp. anatolica P.H. Davis*, Marrubium vulgare L., M. heterodon (Bentham) Boiss. \& Bal.*, **Sideritis sipylea Boiss.*, S. eryhrantha Boiss. \& Heldr. var. eryhrantha**, S. stricta Boiss. \& Heldr**, S. condensata Boiss. \& Heldr. apud.**, S. congesta P.H. Davis \& Hub.Mor.**, S. libanotica Labill. subsp. linearis (Bentham) Bornm.**, S. serratifolia Hub.-Mor.*, Stachys cretica L. subsp. vasillans Rech. fil., S. longispicata Boiss. \& Kotschy*, S. pumilia Banks \& Sol.*, S. antalyensis Y.Ayaşlıgil \& P.H. Davis, S. pseudopinardii Bhattacharjee \& Hub.-Mor.*, S. chasmosericea Ayaşlıgil \& P.H. Davis, $S$. iberica Bieb. subsp. iberica var. densipilosa Bhattacharjee*, S. arvensis (L.) L., **Melissa officinalis L. subsp. officinalis*, Nepeta sulfuriflora P.H. Davis*, N. flavida Hub.-Mor.*, N. pilinux P.H. Davis, N. nuda L. subsp. albiflora (Boiss.) Gams.**, N. caesarea Boiss*, N. cilicia Boiss. apud. Bentham, Lallamentia iberica (Bieb.) Fisch. \& Mey., Prunella vulgaris L.**, P. orientalis Bornm., P. laciniata (L.) L.**, Origanum sipyleum L.**, $O$. minutiflorum O. Schwarz \& P.H. Davis**, **O. majorana L.*, O. onites L.**, O. vulgare L.**, Satureja thymbra L.**, S. cuneifolia Ten**, **Calamintha nepeta (L.) Savi subsp. nepeta*, C. $n$. subsp. glandulosa (Req.) P.W. Ball*, Clinopodium vulgare $\mathrm{L}$. subsp. vulgare**, Acinos rotundifolius Pers., Micromeria nervosa (Desf.) Betham**, M. myrtifolia Boiss. \& Hohen**, M. juliana (L.) Bentham ex Reichb.*, M. graecea (L. ) Bentham ex Reichb. subsp. graecea*, M. elliptica C. Koch., M. cristata (Hampe) Griseb., Thymus cilicicus Boiss. \& Bal.**, T. cherlerioides Vis. var. cherlerioides $* *, * * T$. samius Ronniger. \& Rech. fil.*, **T. zygioides Griseb. var. zygioides*, **T. sibthorpii Bentham.*, **T. longicaulis C. Presl. subsp. longicaulis*, Thymbra spicata L. var. spicata**, **T. s. var. intricata P.H. Davis*, Mentha aquatica L.**, **M. longifolia (L.) Hudson subsp. longifolia*, M. l. subsp. typhoides (Briq.) Harley var. typhoides**, **M. spicata L. subsp. tomentosa (Briq.) Harley*, Lycopus europaeus L., Ziziphora clinopodioides Lam.**, Z. capitata L.**, Salvia tomentosa Miller**, **S. adenophylla Hedge \& Hub.-Mor.*, $S$. 
cadmica Boiss.**, S. sclarea L.**, S. virgata Jacq.**, Ocimum basilicum L.*

Lauraceae

Laurus nobilis L.**

Linaceae

Linum bienne Miller

Loranthaceae

Arceuthobium oxycedri (DC.) Bieb.**, Viscum album L. subsp. austriacum (Wiesb.) Vollman**

Malvaceae

Malva sylvestris L.**, Lavatera thuringiaca L.*, **Alcea calvertii (Boiss.) Boiss.*

Moraceae

**Morus alba L.*, Ficus carica L.**

Myrtaceae

Myrtus communis L. subsp. communis**.

Oleaceae

Jasminum fruticans L.**, Fontanesia philliraeoides Labill. subsp. philliraeoides**, Fraxinus ornus L. subsp. cilicica (Lingels) Yalt.**, **F. angustifolia Vahl. subsp. syriaca*, Phillyrea latifolia L.**

Onagraceae

Epilobium hirsutum L.**, **E. montanum L.*

Orobanchaceae

Orobanche heldreichii (Reuter) G.Beck.*, O. cernua Loefl., O. minor Sm.

Oxalidaceae

**Oxalis acetosella L.*, O. pes-caprae L.*

Paeoniaceae

Paeonia mascula (L.) Miller subsp. mascula**

Papaveraceae

**Glaucium corniculatum (L.) Rud. subsp. corniculatum*, G. leiocarpum Boiss.**, **Papaver apokrinomenon Fedde, **P. strictum Boiss. \& Bal.*, **P. macrostomum Boiss. \& Huet ex Boiss.*, P. rhoeas L.**, **P. postii Fedde, **P. dubium L.*, Hypecoum procumbens L.

Parnassiaceae

Parnassia palustris L.**

Plantaginaceae

Plantago major L. subsp. intermedia (Gilib.) Lange**, P. holosteum Scop., P. lanceolata L.**, P. lagopus L., P. cretica $\mathrm{L}$.

Platanaceae

Platanus orientalis L.**
Plumbaginaceae

Plumbago europea L., Acantholimon confertiflorum Bokhari*

Polygalaceae

Polygala pruinosa Boiss. subsp. pruinosa, P. anatolica Boiss. \& Heldr.

Polygonaceae

Atraphaxis billardieri Jaub. \& Spach. var. billardieri, Polygonum amphibium L.**, P. cognatum Meissn.**, P. arenarium Waldst \& Kit.*, Rumex acetosella L.**, $R$. scutatus L.**, $R$. gracilescens Rech.*, R. patientia L.**, $R$. obtusifolius L. subsp. subalpinus (Schur) Celak.*, $R$. pulcher L.**, R. nepalensis Sprengel.*

Primulaceae

Primula elatior (L.) Hill.**, P. auriculata Lam.**, Androsace maxima L., Cyclamen mirabile Hildebr.**, **C. trachopteranthum O.Schwarz*, Lysimachia atropurpurea L., Anagallis arvensis L. var arvensis*, A. a. var. caerulea (L.) Gouan.

Ranunculaceae

Eranthis hyemalis (L.) Salisb.**, **Nigella sativa L.*, Nigella arvensis L. var. oblanceolata P.H. Davis** Delphinium gueneri P.H. Davis**, Consolida orientalis (Gay) Schröd**,**C. glandulasa (Boiss. \& Huet) Bornm.*, Anemone blanda Schott. \& Kotschy**, A. coronaria L.**, Clematis cirrhosa L.**, Adonis aestivalis L. subsp. aestivalis**, **A. a. subsp. parviflora (Fisch ex DC.) Busch*, **Ranunculus brutius Ten.*, R. neapolitanus Ten, $R$. damascenus Boiss. \& Gaill.*, R. argyreus Boiss., $R$. cuneatus Boiss.*, $R$. reuterianus Boiss., $R$. rumelicus Griseb.*, R. illyricus L., R. $i$. subsp. illyricus, R. cadmicus Boiss., R. heterorhizus Boiss. \& Ball.*, R. isthmicus Boiss. subsp. stepporum Davis, $R$. marginatus d'Urv. var. trachycarpus (Fisch. \& Mey.) Azn., R. scandicinus (Boiss.) Davis*, R. gueneri Ayaşlıgil \& P.H. Davis, R. muricatus L., $R$. chius DC., R. arvensis L., R. ophioglossifolius Vill., $R$. ficaria L. subsp. ficariiformis Rouy \& Fouc.**, Ceratocephalus falcatus (L.) Pers.**, **Thalictrum orientale Boiss.*

\section{Resedaceae \\ Reseda lutea L. var. lutea** \\ Rhamnaceae \\ Paliurus spina-christi Miller**}

\section{Rosaceae}

Prunus x domestica L.**, P. divaricata Ledeb. subsp. divaricata*, **Amygdalus communis L.*, **Rubus caesius L.*, R. sanctus Schreber**, $R$. canescens DC.**, R. c. var.

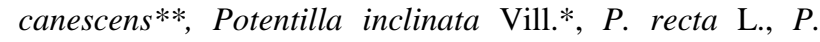
reptans L.**, P. speciosa Willd. var. speciosa*, Geum urbanum L., Agrimonia eupatoria L.**, Sarcopoterium spinosum (L.) Spach**, Sanguisorba minor Scop.**, S. m. subsp. muricata (Spach) Briq., Rosa pulverulenta Bieb.**, $R$. canina L.**, R. heckeliana Tratt. subsp. vanheurekiana (Crepin) Ö. Nilsson*, $R$. villosa L.**, $R$. hirtissima Lonacz**, $R$. hemispherica J.Herrmann**, $\quad$ R. phoenicia Boiss.**, 
$R$. arvensis Huds.**, subsp. boissieri (Crepin)

Rosa

Ö. dumalis Bechst. Ö. Nillson var. antalyensis (Manden) Ö. Nillson**, $R$. gallica L.**, $R$. horrida Fischer**, Cotoneaster nummularia Fisch. \& Mey.**, Crataegus aronia (L.) Bosc. ex DC. var. aronia*, C. monogyna Jacq. subsp. monogyna, C. $m$. subsp. azarella (Gris.) Franco.**, C. orientalis Pall. ex M. Bieb.**, Cydonia oblonga Miller**, Malus sylvestris Mill. subsp. orientalis (A.Uglit) Brow.**, Pyrus syriaca Boiss. var. microphylla Zoh. ex Browicz**, Amelanchier parviflora Boiss.**, Cerasus mahaleb (L.) Miller**

\section{Rubiaceae}

Crucianella angustifolia L.*, Asperula arvensis L., A. setosa Jaub. \& Spach, Galium odoratum (L.) Scop.*, G. debile Desf.*, G. verum L. subsp. verum**, G. album Miller subsp. amani Ehrend. \& Schönb.-Tem.*, G. graecum L. subsp. graecum*, G. dumosum Boiss., G. canum Req. ex DC. subsp. canum*, G. c. subsp. ovatum Ehrend., G. cassium Boiss.*, Cruciata leavipes Opiz.*, C. taurica (Pallas ex Willd.) Ehrend., C. pedemontana (Bellardi) Ehrend., **Rubia tinctorum L.*

Rutaceae

Haplophyllum pumiliforme Hub.-Mor. \& Reese

Salicaceae

Salix alba L.**, Populus tremula L.**, **P. nigra L. subsp. nigra*

\section{Santalaceae}

Thesium bertramii Aznav.*

Scrophulariaceae

Verbascum spodiotrichum (Hub.-Mor.) Hub.-Mor., V. levanticum I.K. Ferguson, V. oreophilum C. Koch*, V. pumiliforme Hub.-Mor., $V$. chrysorrhacos Boiss.*, $V$. purpureum (Janka) Hub.-Mor.*, V. cilicium Boiss.*, V. leiocarpum Murb.*, V. glomerulosum Hub.-Mor., $V$. cucullaribracteum Hub.-Mor.*, Scrophularia cryptophila Boiss. \& Heldr., S. depauperata Boiss., S. canina L. subsp. bicolor (Sm.) Greuter, S. floribunda Boiss. \& Bal.*, Anarrhinum orientale Bentham, Linaria genistifolia (L.) Mill. subsp. confertiflora (Boiss.) Davis**, L. corifolia

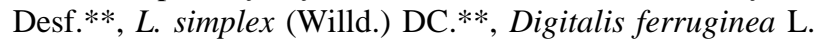
subsp. ferruginea**, D. ferruginea L. subsp. schischkinii (Ivan.) Werner*, D. davisiana Heywood**, Veronica praecox All.*, V. persica Poiret*, V. trichadena Jordan \& Fourr., V. anagallis-aquatica L., V. oxycarpa Boiss., V. jacquinii Baumg.*, V. caespitosa Boiss. var. caespitosa, $V$. macrostachya Vahl subsp. macrostachya*, V. m. subsp. sorgarae M.A. Fischer*, $V$. cuneifolia D.Don subsp. cuneifolia, V. multifida L.*, V. peduncularis Bieb.*, V. serpyllifolia L.*, Lagotis stolonifera (C. Koch) Maxim*, Pedicularis caucasica Bieb.*, P. cadmea Boiss., Rhinanthus angustifolius C.G.Gmelin subsp. grandiflorus (Wallr.) D.A.Webb.*, Rhynchocorys elephans (L.) Griseb. subsp. elephans.

\section{Solanaceae}

Solanum nigrum L. subsp. nigrum**, S. n. subsp. schultesii (Opiz) Wessely, **S. luteum Miller*, Physalis alkekengi L.**, Hyoscyamus niger L.**, H. reticulatus L.**
Styracaceae

Styrax officinalis L.**

Tamaricaceae

**Tamarix parviflora DC.*, **T. smyrnensis Burge*

Thymelaceae

Daphne sericea Vahl.**, D. oleoides Schereber subsp. oleoides*, D. o. subsp. kurdica (Bornm.) Bornm.*, D. gnidioides Jaub. \& Spach.

Tiliaceae

Tilia rubra DC. subsp. caucasica (Rupr.) V. Engler*, T. platyphyllos Scop.**

Ulmaceae

Celtis australis L.**

Urticaceae

Urtica pilulifera L.**, $U$. dioica L.**, Parietaria judaica L., P. lusitanica L.

Valerianaceae

Valeriana oligantha Boiss \& Heldr., V. sisymbriifolia Vahl*, V. officinalis L.*, V. dioscoridis Sm.**, V. cf. dioscoridis Sm., Centranthus calcitrapa (L.) Dufr., Valerianella orientalis (Schlecht) Boiss.\& Bal., V. turgida (Stev.) Betcke

Verbenaceae

Verbena officinalis L.**, Vitex agnus-castus L.**

Violaceae

**Viola odorata L.*, V. heldrechiana Boiss., V. gracilis Sibth. \& Sm.*

Vitaceae
**Vitis vinifera L.*
Zygophyllaceae
Tribulus terrestris L.**, Peganum harmala L.**

Subdivision 2: Liliopsida / Monocotyledoneae

Amaryllidaceae

**Galanthus plicatus Bieb. subsp. byzantinus (Baker) D.A. Webb.*, Pancratium maritimum L.**, Stenbergia lutea (L.) Ker-Gawl. ex Sprengel**

Araceae

Arum maculatum L.**, A. dioscoridis Sm. var. dioscoridis**, A. d. var. liepoldtii (Schott) Engler*

Cyperaceae

Carex cf. vulpinoidea Michaux*, C. otrubae Podp**

Iridaceae

**Iris sintenisii Janka*, Crocus chrysanthus (Herbert) Herbert**, C. biflorus Miller subsp. punctatus Mathew**, C. scharojanii Rupr.*, Gladiolus anatolicus (Boiss.) Stapf.**, **G. atroviolaceus Boiss.* 


\section{Liliaceae}

Smilax aspera L.**, **Ruscus aculeatus L. var. aculeatus*, **Asparagus acutifolius L.2 Polygonatum orientale Desf.**, Asphodelus aestivus Brot.**, Asphodeline lutea (L.) Reichb.**, A. taurica (Pallas) Kunth, A. rigidifolia (Boiss.) Baker, Allium chloranthum Boiss.*, A. bassitense Thieb., A. scorodoprasum L. subsp. rotundum (L.) Stearn, Urginea maritima (L.) Baker**, Scilla bifolia L., S. bithynica Boiss.*, S. autumnalis L., Ornithogalum oligophyllum E.D.Clarke, O. montanum Cyr., **O. nivale Boiss.*, Muscari muscarimi Medicus**, M. comosum (L.) Miller, M. caucasicum (Griseb.) Baker, M. neglectum Guss., Bellevalia bourgaei Baker**, B. rixii Wendelbo*, Fritillaria whittalii Baker**, F. latakiensis Rix**, Tulipa armena Boiss.**, T. a. var. lycica (Baker) Marais**, Gagea bulbifera (Pallas) Schultes \& Schultes fil.*, G. foliosa (J. \& C. Presl) Schultes \& Schultes fil., G. peduncularis (J. \& C. Presl) Pascher, G. granatellii (Parl.) Parl, Colchicum troodii Kotschy**, **Merendera sobolifera C.A. Meyer*, M. trigyna (Steven ex Adam) Stapf**

\section{Orchidaceae}

Cephalenthera rubra (L.) L.C.M. Richard**, Orchis papilionaceae L. var. rubra (Jacq. ex Murray) Brot.**, O. anatolica Boiss.**, O. palustris Jacq.**, **Dactylorhiza romana (Seb.) Soo subsp. romana*, **D. osmanica (K1.) Soo var. osmanica*, D. umbrosa (Kar. \& Kir.) Nevski*

\section{Poaceae (Gramineae)}

Aegilops umbellata Zhukovsky subsp. umbellata**, Ae. triuncialis L. subsp. triuncialis**, **Triticum aestivum L.*, **Secale montanum Guss.*, Hordeum murinum L. subsp. glaucum (Steudel) Tzvelev, H. bulbosum L.**, Bromus hordeaceus L. subsp. hordeaceus**, B. tectorum L.**, B. sterilis L.**,**Avena fatua L. var. fatua*, A. fatua $\mathrm{L}$. var. glabrata Peterm*. Alopecurus myosuroides Hudson var. tonsus (Blanche ex Boiss.) R. Mill., Lolium rigidum Gaudin var. rigidum, L. rigidum Gaudin var. rottbollioides Heldr. ex Boiss., Poa trivialis L.**, **P. bulbosa L., Dactylis glomerata L.. subsp. hispanica (Roth) Nyman**, D. g. subsp. lobata (Drej.) Lindb.*, Briza media L., B. maxima L., B. humilis Bieb., Melica persica Kunth. subsp. inaequiglumis (Boiss.) Bor., Phragmites australis (Cav.) Trin ex Steudel**, Cynodon dactylon (L.) Pers. var. villosus Regel**, Seteria viridis (L.) P. Beauv., S. glauca (L.) P. Beauv., Sorghum halepense (L.) Pers.**

\section{Thyphaceae}

**Typha latifolia L.*

\section{Conclusion}

The national park which located in Western Anatolia has different ecological conditions and high mountainous fields in general. In point of phytogeographical view the area is located in Mediterranean region. It has more primary vegetation more than other areas of Türkiye. It has quite a lot wealth floristically. Isparta is an important province of the Lakes Region which is containing endemic number of taxa about 650, and nonendemic (rare and multiregional) taxa number are almost 1650; totally 2300 vascular plant taxa (Özçelik and Serdaroğlu, 2000). Antalya district is unknown for this aspect. Burdur is poor in flora than Isparta and Antalya provinces. The botanical inventory of Burdur province was made by us; a total of 1600 transmissible plant taxa were identified, including 450 endemic species (Özçelik, 2016). Some of the study area is adjacent to this province.

The vascular plants collected from the areas are divided into 3 groups as follows:

1. Endemics: The plants may be local or widespread endemics in Türkiye,

2. Nonendemics (rare): The plants of narrow dissemination which they found only in small areas. For example, these rare plants are not endemic but were being accepted as distributed only in the Lakes Region for Türkiye.

3. Economics: The plants have economic value like food, dyeing, spices, medicinal etc.

(Ranunculaceae, Brassicaceae, Caryophyllaceae, Lauraceae, Fagaceae, Lamiaceae, Rosaceae, Fabaceae, Papaveraceae, Poaceae, Orchidaceae, Liliaceae, Iridaceae families in particular). These plants are known by local people and are used for domestic needs. Some are endemic and some of them are recorded for the first time for economic purposing. However, they are generally widespread. At least $25 \%$ of plants showing natural distribution in the park are economic purposes. There are two main sources of this information:

Firstly it is the geographical location of the area concerned with respect to the floristic richness and economic plants.

Secondly it is that the experience of nomadism, an ancient tradition of the Turkish people, is still underway by the local people in the area concerned. Even so, even if there is no agricultural production, the local people can meet the needs of plants, such as food, medicine, paint and spices. A significant portion of the cultivated plants have already been obtained from natural plants of the area concerned. It is obvious that these economic plants have an important place in the health of the local people.

The Lakes Region has an important potantiality in terms of economic plants. There are 190 medicinal and aromaticals, 180 foods, 170 horticultural plant taxa in only Isparta province (Özçelik and Serdaroğlu, 2000). A great amount of collected samples from the study area are valuable in medicinal purposes, food, horticulture, dyeing, spices and cultural plants which contribute greatly to the economy of Türkiye. Most of geophytes in Turkiye are known for ornamental values and pharmaceutical purposes. It generally known that Türkiye exports bulbous plants and certain seeds for the horticultural and mainly pharmaceutical purposes. Majority of geophytes and their seeds are taken from the nature by local people (Özçelik, 1995; 2000; 2012; Küçüker, 1995; Ekim et al., 2000). The area was being seen very rich in economic plants.

The study area is part of a long mountain range in the Toros. Other national parks are also connected with this area. Beyşehir Lake National Park, Kovada Lake National Park is the nearest neighbors. Dedegül Mountain is not a national park, but the floristic variety of the Lake District is a very rich mountain. These areas have a similar microclimate. The gene center of rose (Rosa L.) in the world 
is the sum of these areas. R. dumalis subsp. There is boissieri. For antalyaensis, the field type is local and is the gene center (Özçelik, 2017). Again in these areas the Papaver genus develops very well and reaches a high number of varieties.

The same is true for monumental trees. Total number of them in the Lakes Region is about 300 (Özçelik et al., 2001). We guess so, there are about 50 monumental trees beloging to Cupressus sempervirens, Castanea sativa, Cedrus libani, Pinus brutia, P. nigra and Ficus carica, Castanea sativa, as scarcely Myrtus communis, Laurus nobilis, Cercis siliquastrum, Quercus coccifera, Arbutus andrachne and Celtis australis in the park area. It is seem as interesting to be manumental trees from maquis and fruit trees like Castanea sativa, Ficus carica, Myrtus communis, Laurus nobilis, Cercis siliquastrum, Quercus coccifera, Arbutus andrachne and Celtis australis. The region is quite little known floristically. Till now, a large number of new plant taxa for the scientific world were described from the area. It shows a large number of plant taxa which were described from the lakes region and grown only in this area.

Total taxa in dangerous: 48 , for the park, endemic taxa in dangerous: 44, total endemic taxa: 230; local endemic taxa number for the area and near surroundings: 19, total vascular plant taxa: 707 (950 all together near fields).

By Bern Convention: A1 (Number of globally threatened species): 5, A2 (Number of endangered species in European scale): 39. C2 (Number of threatened habitats): 6 (42.A17, 421951, 42B12, 42.6643, 4285B1, 45.11). In the reason, the national park is one of the 122 important plant areas of the country. The situation is very important floristically. If the area is studied in more detail, the number of plants, hence the numbers of endemic and economic plants, will also increase.

Vascular plant taxa endemic to the area (local) are given as below:

Gaudiniopsis macra subsp. micropyroides, Hellenocordum psidicum, Nigella arvensis var. oblanceolata, Rosa dumalis subsp. boissieri var. antalyensis, Scrophularia libanotica subsp. libanotica var. antalyensis, Silene deliculata subsp. pisidica, Stachys antalyensis, Stachys chasmosericea, Tanacetum argenteum subsp. canum var. pumilum, Echinops onopordum, Amphoricarpos paredictus, Bupleurum davisii, Campanula antalyensis, Cerastium pisidicum, Hypericum ternatum, Omphalodes riplyana, Rhamnus nitidus, Iris pamphylica and Crocus asumaniae (this species douptful). Some of these taxa could not observed by us in the area.

Almost 150 taxa are new records to to $\mathrm{C} 3$ square. Some taxa may not be new record in light of new references. In result of the research, the taxa for the square were being done certain and the taxa were added to Isparta and Antalya floras.

A total of 230 endemic taxa belonging to 29 families were determined in the national park and its surroundings. There are 229 taxa belonging to Angiospermae subdivision and 1 taxon belonging to Gymnospermae subdivision. Endemism ratio in flora of the area is almost $25 \% .218$ of the 229 taxa belonging to the Angiospermae are in the Magnoliopsida (Dicotyledoneae) and other 11 are in the Liliopsida Monocotyledoneae) classes. There is no endemic taxon in the Bryophyta and Pteridophyta divisions. The number of priority conservation requiring taxa is 21 . Total taxa in dangerous: 48 , endemic taxa in dangerous: 44 , local endemic taxa for the area and near surroundings: 19, total vascular plant taxa: 707 (950 all together near fields).

Number of genus in vascular plant specimens of the natural park and its environs: Pteridophyta: 8, Gymnospermae: 6, Dicotyledoneae: 318, Monocotyledoneae: 42 and other taxa 333, totally 707 in the park and 950 if included its environs; Number of family: Pteridophyta: 8, Gymnospermae: 3, Dicotyledoneae: 76, Monocotyledoneae: 8 (totally 95 familias).

The distributional rates of the taxa according to phytogeographic regions:

Mediterranean 219 (30.97\%), Irano-Turanian 74 (10.46 $\%)$ and Euro-Siberian 50 (07.07 \%).

The families and genera which including the most taxa:

Families: Lamiaceae 87 (12.30 \%), Fabaceae 78 (11.03 $\%)$, Asteraceae 71 (10.04\%), Brassicaceae 47 (6.64 \%), Scrophulariaceae 39 (5.51\%), Rosaceae 35 (4.95\%), Liliaceae 34 (4.80 \%), Ranunculaceae 33 (\% $4.66 \%)$, Boraginaceae $32(4.52 \%)$, Apiaceae $31(4.38 \%)$ and Caryophyllaceae $28(3.96 \%)$.

Genera: Ranunculus 18 (2.54 \%), Veronica and Geranium 13 (1.83), Vicia 12 (1.69), Trifolium 11 (1.55 \%), Rosa, Verbascum and Sedum 10 (0.14), Galium 9 (1.27), Euphorbia and Stachys 8 (1.13), Sideritis, Hypericum, Trigonella and Astragalus 7 (0.99 \%), Lathyrus, Thymus, Micromeria and Silene $6(0.84 \%)$.

The distributions according to the phytogeographical regions of the endemic plants identified from the area is as follows:

Mediterranean element 219 (30.97\%), Irano-Turanian element $74(10.46 \%)$ and Euro-Siberian element 50 (07.07 $\%)$. Phytogeographical regions of 365 taxa (51.62\%) are unknown. Almost 25 endemic taxa which were known from only type gathering have collected for the first time except for their type localities in the work. For more or less 30 endemic taxa were determined to their which phytogeographical region, for 5 of them changed risk cathegories. Almost 25 endemic taxa which were known from only type gathering have collected for the first time except for their type localities. For more or less 30 endemic taxa were determined to their which phytogeographical region, for 5 of them changed risk cathegories (Ayaşligil, 1987, 1990).

\section{Acknowledgment}

I thank to the research team (Doç.Dr. A. Zafer Tel, Doç.Dr. Ayşe Deligöz, Doç.Dr. Mesut Kırmacı etc.), Antalya GEF II Project Management for interesting and their helps (aspecially Adnan Yılmaztürk, Güray Çayır, Osman Yöntem), TR. Ministry of Forestry and Water Affairs, also the World Bank for financial support in realization of the research project. 


\section{References}

Ayaşlıgil, Y., 1987. Der Köprülü Kanyon National Park, Seine Vegetation und ihre Beeinflussung durch den Menschen. Landschaftsökologie, Weihenstephan, ISBN 3-922 318-05-5.

Ayaşlıgil, Y., 1990. Annonated checklist of vascular flora of Köprülü Canyon National Park and surrouding areas (S.W. Turkey). Willdenowia, vol. 20.

Davis, P.H., 1965-1985. Flora of Turkey and the Aegean Islands. Vol: I-IX, Edinb. Univ. Press.

Donner, J., 1985. Verbreitungsharten zu flora of Turkey. 18. Linzer Biol. Beitr., 17(1): 1-120.

Ekim, T., Koyuncu, M., Vural, M., Duman, H., Aytaç, Z., Adıgüzel, N., 2000. Türkiye Bitkileri Kırmızı Kitabı (Red Data Book of Turkish Plants). Yüzünçü Yıl Üniv. Yayınları, Van.

Fakir, H. 2006a. Bozburun Dağı ve çevresi (AntalyaIsparta) orman vejetasyonunun ana meşcere tiplerinin floristik kompozisyonu üzerine araştırmalar. S.D.Ü. Fen Bilimleri Enstitüsü Dergisi, Cilt:10, Sayı:1,

Fakir, H., 2006b. Flora of Bozburun mountain and its environs (Antalya-Isparta-Burdur/Turkey). Turk J Bot., 30: 149-169.

Güner, A., Özhatay, N., Ekim, T., Başer, K.H.C., 2000. Flora of Turkey and the East Aegean Islands. Vol. 11 (Supplement), Edinburgh Univ. Press.

Huber-Morath, A., 1987. Letzte Erganzungen zu P.H. Davis "Flora of Turkey and the East Aegean Islands" 1-9 (1965-1985). Candollea, 42(2): 717-769.

Huber-Morath, A., 1988. Letzte Erganzungen zu P.H. Davis "Flora of Turkey and the East Aegean Islands" 1-9 (1965-1985)". Candollea, 43: 27-72.

Küçüker, O., 1995. Contributions to the Knowledge of some endangered Colchicum species of Turkey. Flora Mediterranea, 5: 211-220.
Nydegger-Hügli, M., 1994. Sechste Erganzungen zu P.H. Davis "Flora of Turkey and the East Aegean Islands" 110(1965-1988). Bauhinia, 11(2): 103-119.

Özçelik, H., 1995. Studies protections of endemic and rare plants. SDÜ Fen Bilimleri Enst. Derg., 1: 1-14.

Özçelik, H., 2000. Studies on protections of endemic and rare plants of Lakes Region. Bulletin of Pure and Applied Sciences, vol. 19B (No: 2): 93-116.

Özçelik, H., Serdaroğlu, H., 2000. Isparta florasına ön hazırlık. SDÜ Fen Bilimleri Ens. Derg., 4(1): 135-154.

Özçelik, H., Korkmaz, M., Tel, A. Z., Özgökçe, F., 2001. Göller yöresi florası'na genel bakış. IV. Ulusal Ekoloji ve Çevre Kongresi, Bildiriler Kitabı, pp. 117-130.

Özçelik, H., 2012. The endemic plant taxa of the Köprülü Kanyon National Park and its surroundings (AntalyaIsparta). Süleyman Demirel Üniversitesi Fen Bilimleri Enstitüsü Dergisi, 16(3): 279-296.

Özçelik, H., Korkmaz, M., 2002. Contributions to the Flora of Sütçüler (Isparta). Bio-Science Research Bulletin. 21/B (1): 1-18.

Özçelik, H., 2016. Burdur İli Bitki Envanteri (Ekonomik, Nadir ve Endemik Bitkileri). Sistem Ofset, Ankara, Burdur Belediyesi Kültür Yayınları.

Özçelik, 2017. Flora of Dedegül mountain and its effects to agricultural production of Lakes region. International Symposium on New Horizons in Forestry (ISFOR), 1820 October 2017, Isparta - Turkey, Proceedings \& Abstracts Book, pp. 345-353.

Özhatay, N., Byfield, A., Atay, S. (Edit.), 2005. Türkiye'nin 122 Önemli Bitki Alanı. WWF (Türkiye), Doğal Hayatı Koruma Vakfi), İstanbul.

Serin, M., 1984. New Recorded Species for C3 Square. J. of S.Ü. Science Fac., 3: 85-89. 